\title{
Clinico-pathological characteristics of eosinophilic esophagitis in Jordanian children
}

\author{
Eyad Altamimi ${ }^{1}$, Bayan Ahmad ${ }^{1}$, Abdullah Abu-Aqoulah ${ }^{1}$, Naif Rawabdeh ${ }^{2}$ \\ ${ }^{1}$ Paediatric Department, Faculty of Medicine, Jordan University of Science and Technology, Irbid, Jordan \\ 2Paediatric Department, King Abdullah University Hospital, Irbid, Jordan
}

Gastroenterology Rev 2022; 17 (3): 207-212

DOI: https://doi.org/10.5114/pg.2021.109975

Key words: eosinophilic esophagitis, esophageal stenosis, vomiting, dilatation, deglutition disorders.

Address for correspondence: Eyad Altamimi MD, Paediatric Department, Faculty of Medicine, Jordan University of Science and Technology, Irbid, Jordan, e-mail: eyadtamimi@gmail.com

\begin{abstract}
Introduction: Eosinophilic esophagitis (EoE) is a chronic, immune-mediated disorder manifested by symptoms of impaired esophageal function and hypereosinophilic esophageal inflammation. It is believed that the number of patients being diagnosed globally is increasing.

Aim: To describe the frequency and clinicopathological features of EoE in Jordanian children.

Material and methods: A retrospective review of all paediatric patients with eosinophilic esophagitis diagnosed and followed up at our paediatric gastroenterology service at King Abdullah University Hospital between January 2015 and January 2020.

Results: During the study period, 21 patients were diagnosed with EoE out of 700 diagnostic endoscopic procedures. Only $1(4.8 \%)$ female was diagnosed. The average age at presentation was 9.5 years (age range: $1-16$ years). The most common manifestation was solid food dysphagia (15, 71.4\%), followed by food impaction and vomiting $(11,52.4 \%$ and $6,28.6 \%$, respectively). Atopy was diagnosed in 10 (47.6\%) patients. Four (19.0\%) had food allergy, 3 (14.3\%) had respiratory allergy, and $3(14.3 \%)$ had combined. Linear furrows and circular rings were the most common endoscopic features - 17 (81.0\%) and 10 (47.6\%), respectively, while papillary elongation, basal zone hyperplasia, and eosinophilic infiltrate were the most common reported histopathological features. Almost all patients received proton pump inhibitors (19, 90.5\%). Steroids were used in 18 (85.7\%) patients; local steroids in 11 (52.4\%), and oral steroids in 7 (33.3\%). Out of the 4 (19\%) patients with esophageal strictures, $2(9.5 \%)$ required pneumatic dilatation.

Conclusions: Eosinophilic esophagitis is not uncommon in our community. Clinical and histological parameters point to delayed referral and diagnosis. Increasing awareness of this problem will lead to early diagnosis and will decrease complications.
\end{abstract}

\section{Introduction}

Eosinophilic esophagitis (EoE) is characterized by symptoms of impaired esophageal function secondary to chronic, immune-mediated inflammation [1]. Eosinophils are a part of the immune system, and their role in immunity is to fight against specific infections. The accumulation of eosinophils is a marker of allergic diseases [2]. The disorder is characterized by swallowing dysfunctions, such as dysphagia and food impaction, and can cause choking and vomiting. The patient can exhibit some characteristics of gastroesophageal reflux disorder, such as chest pain and food regurgitation [3]. The upper gastrointestinal endoscopy findings include esophageal rings, furrows, exudates, and edema. However, to confirm the diagnosis, a unique histological characteristic of at least 15 eosinophils per high-power field needs to be identified [4].

EoE usually occurs in children and adolescents; however, it may present at any age. In epidemiology, the prevalence in children is estimated at $1 / 2900$ in Europe and North America; however, a fixed increase in the incidence and prevalence is observed. Boys are mostly affected (male/female ratio of 2.5) [5]. A higher prevalence of EoE is found in Europe and North America, compared to Eastern countries, which may indicate the influence of environmental and immune factors [6]. The management of EoE revolves around controlling the symptoms, preventing disease progression, improving the quality of life, and reversing complications [7]. The cornerstones of management are proton pump inhibi- 
tors, local steroids, dietary restriction, and esophageal dilatation in case of esophageal strictures [8].

With respect to Arab countries, a previous publication from Egypt reported a low prevalence of EoE (1.87\%) among adults who had undergone upper endoscopy [9]. Although a report from Western Saudi Arabia found a $0.85 \%$ prevalence of EoE among children who had undergone upper endoscopy [10], Saeed et al. reported a prevalence of up to $9.3 \%$ among children who had undergone upper endoscopy in Riyadh [11].

Data on EoE from Jordan are scarce. A previous report from Jordan described 38 patients with a histological diagnosis of eosinophilic esophagitis. The majority of the patients were boys. The authors concluded that EoE in Jordan had clinical, endoscopic, and pathological features similar to those described in other countries [12]

\section{Aim}

The aim of the study is to describe the relative frequency and clinicopathological characteristics of patients with EoE diagnosed and managed at our paediatric gastroenterology service at a tertiary hospital in North Jordan.

\section{Material and methods}

This was a retrospective observational study review of all the children with eosinophilic esophagitis diagnosed and followed up at our paediatric gastroenterology service at King Abdullah University Hospital between January 2015 and January 2020.

Patients were identified using the electronic medical records of the hospital, endoscopy database, and pathology records.

Data regarding the age, sex, age at presentation, presenting symptoms, physical findings, atopy, endoscopic and histopathological findings, treatment offered, and the outcomes were retrieved.

Exclusion criteria: patients with no histopathological data, patients with eosinophilic count < 15 per high-power field in esophageal biopsies, patients aged $>18$ years at diagnosis.

All the patients underwent an upper endoscopy. All the procedures were completed by a senior paediatric gastroenterologist in the paediatric endoscopy unit at King Abdullah University Hospital. Our sedation protocol includes light sedation with intravenous midazolam and local lidocaine spray. Heavier sedation (ketamine/ fentanyl) was used on a case-specific basis. Multiple esophageal biopsies were performed at different levels.

Histopathological assessments were performed in the histopathology department at King Abdullah University Hospital by a senior pathologist, paediatric pathologist, or gastrointestinal pathologist.

\section{Ethical analysis}

This study was approved by the IRB committee of the Faculty of Medicine and Research Committee of the Jordan University of Science and Technology (2020-764).

\section{Statistical analysis}

Data were collected and entered into an Excel sheet. Data are presented as averages and percentages.

\section{Results}

Of the 700 diagnostic upper endoscopy procedures performed during the study period, 21 patients with EoE were identified. The relative frequency was $3 \%$. Twenty (95.2\%) patients were boys. The average age was 9.4 years ( $1-16$ years) (Table I).

The most common presenting symptom was solid food dysphagia in 15 (71.4\%) patients, followed by food impaction in 11 (52.4\%), and recurrent vomiting in $6(28.6 \%)$. A history of allergic disorders was reported in $10(47.6 \%)$ patients, food allergies in 4 (19\%), respiratory allergies in 3 (14.3\%), and both food and respiratory allergies in 3 (Table I).

All patients underwent an upper endoscopy. The most common endoscopic finding was linear furrows in 17 (81\%) patients, followed by circular esophageal rings and whitish papules in 10 (47.6\%) patients. Endoscopic examination of the esophagus was reported to be normal in 2 patients. Four (19\%) patients had esophageal strictures. Concomitant $H$. pylori infection was diagnosed in 5 (23.8\%) patients by the histopathological examination of gastric biopsies (Table II).

Four (19\%) patients had asthma. The allergy work-up showed peripheral eosinophilia (Eos. count $>500)$ in $5(23.8 \%)$ patients and hyper immunoglobin (Ig) E (IgE level > 100) in 6 (26.8\%) patients. All the patients underwent a radioallergosorbent (RAST) test, with 7 (33.3\%) patients testing positive for anti-egg yolk antibody, and 3 for anti-milk antibody (42.9\%) (Table III).

Almost all the patients received proton pump inhibitors (PPIs) ( $n=19,90.5 \%)$. Steroids were used in 18 (85.7\%) patients, including local steroids $11 / 21$ ) and oral steroids (7/21). PPIs were received by 19 (90.5\%) patients, inhaled steroids by 13 , and oral budesonide by 6 . Two (9.5\%) patients had severe strictures that did not respond to medical treatment and required balloon dilatation. Although the patients (5 patients) were advised food elimination, only 3 families agreed to this. Upon follow-up, most of the patients showed clinical improvement, 8 patients underwent repeated endoscopy, and only 2 of the patients showed complete histological resolution. 
Table I. Demographic data and clinical manifestations in eosinophilic esophagitis (EoE)

\begin{tabular}{|c|c|}
\hline Parameter & Value \\
\hline Total number of patients, $n$ (\%) & $21(100)$ \\
\hline Male, $n(\%)$ & $20(95.2)$ \\
\hline Female, $n(\%)$ & $1(4.8)$ \\
\hline Age [years] & 9.4 \\
\hline Age range [years] & $1-16$ \\
\hline History of allergy, $n$ (\%): & $10(47.6)$ \\
\hline Food allergy & $4(19)$ \\
\hline Respiratory allergy & $3(14.3)$ \\
\hline Both & $3(14.3)$ \\
\hline Family history of atopy, $n$ (\%) & $1(4.8)$ \\
\hline \multicolumn{2}{|l|}{ Presenting symptoms, $n(\%)^{\star}$ : } \\
\hline Solid food dysphagia & $15(71.4)$ \\
\hline Food impaction & $11(52.4)$ \\
\hline Vomiting & $6(28.6)$ \\
\hline Cough & $5(23.8)$ \\
\hline Heartburn & $3(14.3)$ \\
\hline Regurgitation & $2(9.5)$ \\
\hline Food refusal & $2(9.5)$ \\
\hline Non-swallowing chest pain & $2(9.5)$ \\
\hline Failure to thrive & $1(4.8)$ \\
\hline Nausea & $1(4.8)$ \\
\hline Abdominal pain & $1(4.8)$ \\
\hline Odynophagia & $1(4.8)$ \\
\hline
\end{tabular}

*Numbers might not add up because some patients might have more than 1 presenting symptom.

\section{Discussion}

EoE is a chronic immune-mediated disorder characterized by clinicopathological findings related to esophageal dysfunction clinically and eosinophil-predominant inflammation histologically [13]. It has been commonly reported in the pediatric population; however, it has recently been increasingly recognized in the adult population $[12,14]$. Although there has been an increase in the incidence of EoE cases in both pediatric and adult populations, there have been few studies conducted in Jordan $[12,15,16]$. In our study, we aimed to provide more data about EoE in Jordanian children, its relative frequency, and its clinicopathological characteristics.

It is believed that the incidence and prevalence of EoE are increasing worldwide. Despite the geographical variations, a consistent increase in the diagnosis is still recognized. Population-based studies have shown
Table II. Endoscopic and histological findings

\begin{tabular}{lc} 
Endoscopy and histology findings & Value, $\boldsymbol{n}(\%)$ \\
\hline Linear furrows & $17(81)$ \\
\hline Esophageal rings & $10(47.6)$ \\
\hline Whitish papules & $10(47.6)$ \\
\hline Stricture & $4(19)$ \\
\hline Papillary elongation & $10(47.6)$ \\
\hline Eosinophil surface layering & $7(33.3)$ \\
\hline Basal zone hyperplasia & $7(33.3)$ \\
\hline eosinophilic microabscess & $5(23.8)$ \\
\hline Esophageal ulcerations & $6(28.6)$ \\
\hline Dilated intracellular space & $4(19)$ \\
\hline Mucosal friability & $2(9.5)$ \\
\hline Normal esophagus & $2(9.5)$ \\
\hline Lamina propria fibrosis & $2(9.5)$
\end{tabular}

Table III. Allergic work-up results

\begin{tabular}{lc} 
Variable & Results \\
\hline Allergy tests (RAST) (positive): & \\
\hline Egg & 5 \\
\hline Milk & 3 \\
\hline Mango & 1 \\
\hline Peanut & 1 \\
\hline Blood test: & $5(23.8 \%)$ \\
\hline Eosinophilia $n(>500 / \mu l)$ & $6(28.6 \%)$ \\
\hline Hyper lgE $n(>100 \mathrm{IU} / \mathrm{ml})$ & $12.7(9.7-15.6)$
\end{tabular}

a steady increase over the past 2 decades in North America, Europe, and Australia [17].

Although the comparison may not be entirely scientific due to the time reference and the diagnostic criteria used, our estimated EoE prevalence in all the patients who underwent endoscopy was 3\%, which lies between the Turkish [18] estimates and the reports from Saudi Arabia [19]. It has been observed that the number of cases is increasing in Jordan. A previous study from central Saudi Arabia estimated the relative frequency of EoE in children who underwent upper endoscopy for any reason at $4.8 \%$ [19]. A newer report from the same group 5 years later reported a 2-fold rate of approximately $9.3 \%$ [12].

Although our study was not a true epidemiological study and there are no previous numbers for comparison, when comparing our numbers (21 cases in 5 years) 
to the number of diagnosed cases in another referral centre in Jordan with a higher endoscopy service volume [12] (38 cases over 10 years), the incidence may be increasing.

EoE is reported more frequently in men than in women. Approximately $60-80 \%$ of all the cases were diagnosed in men. The analysis of studies with detailed demographic information of adult patients has shown that, on average, $76 \%$ of those affected were men, thereby suggesting a male-to-female risk ratio of $3: 1$ [20]. Genta et al. [21] demonstrated that a male predominance persisted in a variety of ethnic groups in the United States, suggesting that genetic factors may play a role. Notably, this effect was most prominent among Arabs. A single nucleotide polymorphism (SNP) in the thymic stromal lymphopoietin (TSLP) gene and nonsynonymous SNPs in the TSLP receptor is hypothesized to be associated with EoE only in male patients [22]. It is notable that this has not been studied in Arabs. In our study, the higher prevalence in men among patients with EoE was similar to that previously reported by Arabs and other countries [10, 12, 19, 21, 23].

EoE is an allergic condition, and allergic triggers that provoke the disease have been implicated. Concomitant allergic conditions, such as bronchial asthma, allergic rhinitis, and food allergies, are commonly reported in patients with EoE [24]. A study was conducted in Philadelphia, Pennsylvania, in which patients (14\%) with EoE were suggested to have aeroallergen-associated triggers by history. Among these 160 patients, 32 (20\%) had a biopsy examination-confirmed variant of EoE triggered by aeroallergens. Most of these patients were boys (84\%), all had a history or examination consistent with allergic rhinitis, and most had a history of asthma (75\%). A total of 32 patients exhibited obvious seasonal variation, 22 (13\%) of whom also had known food-induced symptoms [25]. Notably, 59\% of children with EoE and IgE-mediated food allergies were reported in Colorado, USA [26], compared with $29 \%$ of children enrolled in a multicentre European study [27]. In a Polish study, the patients' history analysis revealed a higher percentage of allergy cases in children with EoE, with seasonal allergies at $23.08 \%$ and food allergies at $11.1 \%$ [23]. In a study from Saudi Arabia, $50 \%$ of the patients had atopy [10]. A previous report from Jordan reported food allergies according to the RAST in 39\% and bronchial asthma in $23 \%$ of patients. Our results were consistent with those of previous reports showing that almost half of the patients had allergic conditions. Notably, egg allergy was the most commonly diagnosed food allergy in our cohort.

The clinical manifestations of EoE are age dependent. Although feeding issues, namely vomiting, are prevalent in the younger age groups, dysphagia, food impaction, and abdominal pain are the presenting symptoms in older children [24]. In our cohort, solid food dysphagia was the most common presenting symptom (71.4\%). Our results were somewhat consistent with those from a report from Saudi Arabia [10]; however, a study from Jordan reported that vomiting was the most common presenting symptom. This may be explained by the fact that our patients were older than those in the KHMC cohort [12].

Food impaction is mostly observed in the adult EoE. Previous studies from Poland, New Zealand, and Brazil reported that none of their EoE children had food impaction [23, 28, 29]. In contrast, Ristic et al. [30] reported that $36 \%$ of the children with EoE presented with food impaction. In our cohort, approximately half of the patients presented with food impaction. This may reflect a delayed referral and the development of complications related to longstanding disease.

Notably, although there were gastrointestinal symptoms and feeding issues, none of our patients developed a failure to thrive. This is consistent with previous reports by Mehta et al. [26] and Zdanowicz et al. [23]. We do not believe that this is due to the benign nature of the disease; however, this may reflect the adaptive behaviour of the patients and their parents.

The diagnosis of EoE is dependent on the coupling characteristics of endoscopic and histopathological findings. The universal macroscopic findings are linear furrows, whitish papules, and stricturing, whereas microscopically, in addition to the esophageal eosinophilia, basal cell hyperplasia and eosinophilic abscesses can be found [28, 30-32]. The most common endoscopic finding was linear furrows, followed by esophageal rings and whitish spots. Notably, 2 (9.5\%) patients showed normal endoscopic examination results. This is still lower than what has been reported in the US [33], New Zealand [28], and Brazil [29]. We believe that this may reflect a delay in the referral and diagnosis of our cohort.

Helicobacter pylori infection has been inversely associated with allergic diseases, including asthma, atopic dermatitis, and allergic rhinitis, and $H$. pylori may play a protective role in these conditions [34]. Von Armin et al. showed an inverse relationship between $H$. pylori infection and EoE development [35]. In contrast, Molina-Infante et al. in their multicentre case-control study in Europe did not demonstrate this effect [36]. Zdanowicz et al. [23] found similar rates of $H$. pylori infection in both the EoE and non-EoE groups. H. pylori infects approximately $15 \%$ of Jordanian children [37]. In our cohort, $23.8 \%$ had biopsy-proven $H$. pylori infection. Although this adds to the conflicting data on the role 
of $H$. pylori in EoE, our data did not support the role of H. pylori in EoE development.

EoE requires repeated endoscopic biopsies for diagnosis and monitoring. Reliable non-invasive markers are yet to be identified [38]. The most commonly reported biomarkers are peripheral blood eosinophils. Although the absolute eosinophilic count has been suggested to serve as a marker of disease activity, it still needs further development. In our cohort, only $30 \%$ of the patients showed peripheral blood eosinophilia. This was lower than the reported rates, even in Jordan. This may support the conclusion of Hines et al. [38] that further work is needed to develop non-invasive markers for EoE.

Eosinophilic esophagitis is a chronic immune-mediated disorder with significant morbidity. The goals of treatment are alleviating symptoms, improving the quality of life, and improving microscopic healing [7]. PPIs, swallowed topical/oral steroids, and food elimination are the therapeutic options available [24]. Oral viscous steroids provide higher levels of esophageal contact time with the drug, which is why they are preferred over metered dose inhalers [39]. Almost all our patients started on PPIs. This was followed by administration of local steroids. The elimination diet was the most difficult to follow. Three patients only agreed to 6 food elimination diets, and only 1 patient was compliant. Although viscous budesonide is not commercially available in Jordan, home-prepared formulary by adding budesonide inhalation solution to honey was used effectively.

Clinical improvement alone is insufficient and needs to be coupled with histological assessment [40]. Although most of our patients had improved symptoms, $8(38.1 \%)$ underwent repeated endoscopy, and only 2 patients had normalized histology. Long-standing untreated disease leads to subepithelial fibrosis, narrow esophagus, and stricture formation [41]. In our cohort, 4 patients had esophageal strictures, and 2 of them required pneumatic dilatation, which is consistent with a previous report from Jordan.

This is the first report from North Jordan presenting data on the clinicopathological features of EoE from Jordan. This study had several limitations. First, this was a retrospective study. The small number of enrolled patients restricts the generalization of our results. The unavailability of other advanced testing methods, such as $\mathrm{pH}$ monitoring and skin prick testing, limits the comparability of our results with some published literature.

\section{Acknowledgments}

The authors would like to thank the staff of the endoscopy unit at King Abdullah University Hospital for their help and professionalism.

\section{Conflict of interest}

The authors declare no conflict of interest.

\section{References}

1. Reed CC, Dellon ES. Eosinophilic esophagitis. Med Clin North Am 2019; 103: 29-42.

2. Klion AD, Rothenberg ME. Advances in eosinophilic diseases in 2018. J Allergy Clin Immunol 2019; 144: 1490-4.

3. Straumann A. Eosinophilic esophagitis: rapidly emerging disorder. Swiss Med Wkly 2012; 142: w13513.

4. Surdea-Blaga T, Popovici E, Fadgyas Stănculete M, et al. Eosinophilic esophagitis: diagnosis and current management. J Gastrointestin Liver Dis 2020; 29: 85-97.

5. Katzka DA. Eosinophilic esophagitis. Gastrointest Endosc Clin N Am 2018; 28: 1-14.

6. Gómez-Aldana A, Jaramillo-Santos M, Delgado A, et al. Eosinophilic esophagitis: current concepts in diagnosis and treatment. World J Gastroenterol 2019; 25: 4598-613.

7. Sodikoff J, Hirano I. Therapeutic strategies in eosinophilic esophagitis: induction, maintenance and refractory disease. Best Pract Res Clin Gastroenterol 2015; 29: 829-39.

8. Vinit C, Dieme A, Courbage S, et al. Eosinophilic esophagitis: pathophysiology, diagnosis, and management. Arch Pediatr 2019; 26: 182-90.

9. Fouad M, Fouad YM, Mokareb HA, et al. Prevalence of eosinophilic esophagitis in adult patients with upper gastrointestinal symptoms in a locality in upper Egypt. Clin Endosc 2018; 51: 357-61.

10. Saadah OI, Aburiziza AJ, Abu Shakra RI. Eosinophilic esophagitis in children from Western saudi arabia: relative frequency, clinical, pathological, endoscopic, and immunological study. Gastroenterol Res Pract 2012; 2012: 328253.

11. Saeed A, Assiri AM, Al Asmi M, et al. Trend, clinical presentations and diagnosis of eosinophilic esophagitis in Saudi children. Saudi Med J 2018; 39: 668-73.

12. Al-Bderat J, Ghanma A, Ruabdeh S, et al. Eosinophilic esophagitis among children at King Hussein Medical Center. J R Medic Serv 2012; 20: 48-52.

13. Liacouras CA, Furuta GT, Hirano I, et al. Eosinophilic esophagitis: updated consensus recommendations for children and adults. J Allergy Clin Immunol 2011; 128: 3-20.e6.

14. Arora AS, Yamazaki K. Eosinophilic esophagitis: asthma of the esophagus? Clin Gastroenterol Hepatol 2004; 2: 523-30.

15. Al-Hussaini AA, Semaan T, El Hag IA. Esophageal trachealization: a feature of eosinophilic esophagitis. Saudi J Gastroenterol 2009; 15: 193-5.

16. Hasosah MY, Sukkar GA, Alsahafi AF, et al. Eosinophilic esophagitis in Saudi children: symptoms, histology and endoscopy results. Saudi J Gastroenterol 2011; 17: 119-23.

17. Dellon ES, Hirano I. Epidemiology and natural history of eosinophilic esophagitis. Gastroenterology 2018; 154: 319-32.e3.

18. Altun R, Akbas E, Yıldırım AE, et al. Frequency of eosinophilic esophagitis in patients with esophageal symptoms: a single-center Turkish experience. Dis Esophagus 2013; 26: 776-81.

19. Assiri AM, Saeed A. Incidence and diagnostic features of eosinophilic esophagitis in a group of children with dysphagia and gastroesophageal reflux disease. Saudi Med J 2014; 35: 292-7. 
20. Hruz P. Epidemiology of eosinophilic esophagitis. Dig Dis 2014 32: $40-7$

21. Genta R, Turner K. Eosinophilic esophagitis: male predominance is present across all ethnic groups. Am J Gastroenterol 2016; 111: S229-30.

22. Sherrill JD, Gao PS, Stucke EM, et al. Variants of thymic stromal lymphopoietin and its receptor associate with eosinophilic esophagitis. J Allergy Clin Immunol 2010; 126: 160-5.e3.

23. Zdanowicz K, Kucharska M, Sobaniec-Lotowska ME, et al. Eosinophilic esophagitis in children in North-Eastern Poland. J Clin Med 2020; 9: 3869.

24. Lucendo AJ, Molina-Infante J, Arias Á, et al. Guidelines on eosinophilic esophagitis: evidence-based statements and recommendations for diagnosis and management in children and adults. United European Gastroenterol J 2017; 5: 335-58.

25. Ram G, Lee J, Ott M, et al. Seasonal exacerbation of esophageal eosinophilia in children with eosinophilic esophagitis and allergic rhinitis. Ann Allergy Asthma Immunol 2015; 115: 224-8.

26. Mehta P, Furuta GT, Brennan T, et al. Nutritional state and feeding behaviors of children with eosinophilic esophagitis and gastroesophageal reflux disease. J Pediatr Gastroenterol Nutr 2018; 66: 603-8

27. Hoofien A, Dias JA, Malamisura M, et al. Pediatric eosinophilic esophagitis: results of the European Retrospective Pediatric Eosinophilic Esophagitis Registry (RetroPEER). J Pediatr Gastroenterol Nutr 2019; 68: 552-8.

28. Weerasekera K, Sim D, Coughlan F, et al. Eosinophilic esophagitis incidence in New Zealand: high but not increasing. Clin Exp Gastroenterol 2019; 12: 367-74.

29. Pinheiro MI, de Góes Cavalcanti LP, Honório RS, et al. Eosinophilic esophagitis in Brazilian pediatric patients. Clin Med Insights Pediatr 2013; 7: 41-8.

30. Ristic N, Jankovic R, Dragutinovic N, et al. Diagnosis of eosinophilic esophagitis in children: a Serbian single-center experience from 2010 to 2017. Med Princ Pract 2019; 28: 449-56.

31. Tan LN, Srivastava S, Teh M, et al. Eosinophilic oesophagitis in children: an uncommon occurrence in a predominantly Chinese population in Singapore. Singapore Med J 2017; 58: 218-22.

32. Aceves SS, Newbury RO, Dohil R, et al. Distinguishing eosinophilic esophagitis in pediatric patients: clinical, endoscopic, and histologic features of an emerging disorder. J Clin Gastroenterol 2007; 41: 252-6.

33. Liacouras CA, Spergel JM, Ruchelli E, et al. Eosinophilic esophagitis: a 10-year experience in 381 children. Clin Gastroenterol Hepatol 2005; 3: 1198-206

34. Chen Y, Blaser MJ. Helicobacter pylori colonization is inversely associated with childhood asthma. J Infect Dis 2008; 198: 553-60.

35. von Arnim U, Wex T, Link A, et al. Helicobacter pylori infection is associated with a reduced risk of developing eosinophilic oesophagitis. Aliment Pharmacol Ther 2016; 43: 825-30.

36. Molina-Infante J, Gutierrez-Junquera C, Savarino E, et al. Helicobacter pylori infection does not protect against eosinophilic esophagitis: results from a large multicenter case-control study. Am J Gastroenterol 2018; 113: 972-9.
37. Altamimi E, Alsharkhat N, AlJawarneh A, et al. Declining prevalence of Helicobacter pylori infection in Jordanian children, report from developing country. Heliyon 2020; 6: e04416.

38. Hines BT, Rank MA, Wright BL, et al. Minimally invasive biomarker studies in eosinophilic esophagitis: a systematic review. Ann Allergy Asthma Immunol 2018; 121: 218-28.

39. Dellon ES, Sheikh A, Speck O, et al. Viscous topical is more effective than nebulized steroid therapy for patients with eosinophilic esophagitis. Gastroenterology 2012; 143: 321-4.e1.

40. Munoz-Persy M, Lucendo AJ. Treatment of eosinophilic esophagitis in the pediatric patient: an evidence-based approach. Eur J Pediatr 2018; 177: 649-63.

41. Robles-Medranda C, Villard F, le Gall C, et al. Severe dysphagia in children with eosinophilic esophagitis and esophageal stricture: an indication for balloon dilation? J Pediatr Gastroenterol Nutr 2010; 50: 516-20.

Received: 4.07.2021

Accepted: 28.07.2021 